\title{
STRUCTURAL STABILITY AND HYPERBOLIC ATTRACTORS
}

\author{
BY
}

\section{ARTUR OSCAR LOPES}

\begin{abstract}
A necessary condition for structural stability is presented that in the two dimensional case means that the system has a finite number of topological attractors.
\end{abstract}

Introduction. One of the basic questions in Dynamical System Theory is the characterization and the study of the properties of structurally stable systems (vector fields or diffeomorphisms). The standing conjecture about characterization, first formulated by Palis and Smale [2], is that a system is structurally stable if and only if it satisfies Axiom A and the strong transversality condition. That these conditions are sufficient was proved by Robbin [8] and Robinson [9], [10] after a series of important results in this direction. It is also known that in the presence of Axiom A, strong transversality is a necessary condition for stability. Therefore the open question is whether stability implies Axiom A. Relevant partial results were obtained by Mañé [3], [4] and Pliss [5], [6]. Pliss [5] showed that $C^{1}$-stability of a diffeomorphism implies that there is only a finite number of attracting periodic orbits (all hyperbolic). The purpose of this work is to show, generalizing this last result, that $C^{1}$-structurally stability of a diffeomorphism implies that there is only a finite number of hyperbolic attractors. This result is a well known fact for Axiom A diffeomorphisms (Smale [11]).

We will now explain some facts about our result.

Let $M$ be a $C^{\infty}$-manifold without boundary and $\operatorname{Diff}^{r}(M)$ be the space of $C^{r}$-diffeomorphisms of $M$ with the $C^{r}$-topology, $r>1$. A diffeomorphism $f \in \operatorname{Diff}^{r}(M)$ is $C^{r}$-structurally stable if there exists a neighborhood $U$ of $f$ in $\operatorname{Diff}^{r}(M)$, such that if $g \in U$ there exist a homeomorphism $h$ of $M$ satisfying $f h=h g$. Such a diffeomorphism $f$ has the property that all diffeomorphisms sufficiently near to it have the same orbit structure.

We will call a point $x \in M$ nonwandering for $f$ when $\forall U$ neighborhood of $x$ in $M$ there exist $n \in \mathbf{Z}-\{0\}$, such that $f^{n}(U) \cap U \neq \varnothing$. We will denote such a set by $\Omega(f)$.

A closed subset $\Lambda$ of the nonwandering set $\Omega(f)$ is called an attractor when:

(1) $\Lambda$ is $f$-invariant (that is, $f(\Lambda)=\Lambda$ ).

Received by the editors April 4, 1978.

AMS (MOS) subject classifications (1970). Primary 58F10; Secondary 58F15.

Key words and phrases. Structural stability, Axiom A, hyperbolic set, attractor, stable manifold. 
(2) $\exists L$ neighborhood of $\Lambda$ in $M$ such that $\bigcap_{n \in \mathbf{Z}_{+}} f^{n}(L)=\Lambda$.

(3) $f$ is transitive.

A subset $\Delta \subset M$ is called hyperbolic for a diffeomorphism $f \in \operatorname{Diff}^{r}(M)$ when

(1) $\Delta$ is $f$-invariant.

(2) There exist continuous bundles $E_{s}(x)$ and $E_{u}(x)(x \in \Delta)$ that are invariant by $D f$ and such that $E_{s}(x) \oplus E_{u}(x)=T M_{x}, \forall x \in \Delta$.

(3) $\exists K>0, \exists \lambda, 0<\lambda<1$, such that

$$
\begin{aligned}
\left\|(D f)^{n} \mid E_{s}(x)\right\| & \leqslant K \lambda^{n}, \\
\left\|(D f)^{-n} \mid E_{u}(x)\right\| & \leqslant K \lambda^{n} \quad \forall x \in \Delta, \forall n \in \mathbf{Z}_{+} .
\end{aligned}
$$

Our purpose in this work is to prove the following:

THEOREM. The number of hyperbolic attractors in a $C^{1}$-structurally stable diffeomorphism is finite.

In the study of $C^{r}$-structurally stable diffeomorphisms the following definitions were introduced (Smale [11]):

Axıom A. We will say that a diffeomorphism $f \in \operatorname{Diff}^{r}(M)$ satisfies the Axiom A when:

(1) the periodic points are dense in the nonwandering set,

(2) the nonwandering set of the diffeomorphism $f$ is hyperbolic.

Stable (RESPECTIVEly UNSTABle) MANIFOld. For a diffeomorphism $f \in$ $\operatorname{Diff}^{r}(M)$ and a point $x \in M$ we will call stable (unstable) manifold of $x$ the following set: $W^{s}(x)=\left\{y \in M \mid d_{n \rightarrow \infty}\left(f^{n}(x), f^{n}(y)\right) \rightarrow 0\right\} \quad\left(W^{u}(x)=\{y \in\right.$ $\left.\left.M \mid d_{n \rightarrow \infty}\left(f^{-n}(x), f^{-n}(y)\right) \rightarrow 0\right\}\right)$.

It can be proved [1] that if $f$ satisfies Axiom $A$ then for any $x \in \Omega(f)$, $W^{s}(x)\left(W^{u}(x)\right)$ is a 1-1 immersed manifold in $M$ and

$$
\bigcup_{x \in \Omega(f)} W^{s}(x)=\bigcup_{x \in \Omega(f)} W^{u}(x)=M
$$

STRONG TRANSVERSALITY. We will say that a diffeomorphism $f \in \operatorname{Diff}^{r}(M)$ satisfies the strong transversality condition when:

(1) $f$ satisfies Axiom A,

(2) the intersections between stable and unstable manifolds of any pair of points in the nonwandering set are transversal.

In the direction of the characterization of the $C^{r}$-structurally stable diffeomorphisms there exists the following conjecture:

Conjecture 1. A diffeomorphism $f \in \operatorname{Diff}^{r}(M)$ satisfies the Axiom A and the strong transversality condition if and only if it is $C^{r}$-structurally stable.

It is a well known fact (Smale [11]) that a diffeomorphism satisfying Axiom $\mathrm{A}$ and strong transversality conditon has a finite number of hyperbolic 
attractors. A complete proof of this conjecture would therefore contain our result.

We point out that from Mañe [4] it follows that if $\Lambda$ is an attractor of a diffeomorphism $C^{1}$-structurally stable in a manifold of dimension two, then $\Lambda$ is hyperbolic. Therefore in dimension two our main result can be stated in the following way. The number of attractors in a $C^{1}$-structurally stable diffeomorphism is finite.

The proof of our result will be done in the following way: in $\$ 1$ we relate and explain some known facts, mainly from [4] and [5]. In \$2 we demonstrate some preliminary lemmas that we will lead in $\$ 3$ to our objective.

This paper is the author's doctoral thesis at IMPA under the guidance of $J$. Palis. I wish to thank him and R. Mañé for many helpful conversations.

1. For Axiom A diffeomorphisms there exists a generalization of stable (unstable) manifolds for periodic points to the points of the nonwandering set [1]. This generalization allows us to deal with a continuous family of discs that have some interesting properties. We will call this family local stable (unstable) manifolds and we will enumerate some of its properties in Lemmas 1.1 and 1.2 .

For $C^{1}$-structurally stable systems we do not have "a priori" this nice structure of discs, that are defined by the hyperbolicity of the nonwandering set. However another continuous family of discs was introduced by Mañé [4] for $C^{1}$-structurally stable diffeomorphisms, that has some but not all properties of the family of local stable (unstable) manifolds. We will call this new family of discs the family of pseudo-stable (pseudo-unstable) manifolds. We notice that these two kind of families stable and pseudo-stable (unstable and pseudo-unstable) coincide in the case where $f$ satisfies Axiom A.

In this section we will remember some of the properties of this two families. In Lemmas 1.1 and 1.2 we will talk about stable (unstable) manifolds, in the hyperbolic case. In Lemmas 1.3 through 1.8 we will be interested in the pseudo-stable (pseudo-unstable) manifolds of a $C^{1}$-structurally stable diffeomorphism. We will just give the proofs of Lemmas 1.5 and 1.8 because the others are proved in [4].

In Lemma 1.9 we relate the two splittings that are associated with these two families in the case of a hyperbolic attractor of a $C^{1}$-structurally stable diffeomorphism.

Finally in Lemma 1.11 we will discuss the use of techniques introduced by Pliss in [5] applied to the pseudo-stable manifolds of a $C^{1}$-structurally stable diffeomorphism.

We will give before Lemma 1.11 an idea of where we will use all this machinery to prove the main result of this work.

In the rest of this work we will consider a $C^{\infty}$ compact $n$-dimensional 
manifold $M$ without boundary and $\operatorname{Diff}^{1}(M)$ the set of diffeomorphisms of $M$ with the $C^{1}$-topology. We will consider $d($,$) a fixed metric on M$.

LeMMA 1.1. For $x$ in a hyperbolic set $\Lambda$ with the periodic points dense in $\Lambda$, there exists an unstable (respectively stable) manifold of $x$ denote by $W^{u}(x)$ $\left(W^{s}(x)\right)$ with the following properties:

(a) $W^{u}(x)=\left\{y \in M \mid d_{n \rightarrow \infty}\left(f^{-n}(x), f^{-n}(y)\right) \rightarrow 0\right\} \quad\left(W^{s}(x)=\{y \in\right.$ $\left.\left.M \mid d_{n \rightarrow \infty}\left(f^{n}(x), f^{n}(y)\right) \rightarrow 0\right\}\right)$ is a 1-1 immersed plane with the same dimension as $E^{u}(x)\left(E^{s}(x)\right)$,

(b) $E^{u}(x)\left(E^{s}(x)\right)$ is the tangent plane of $W^{u}(x)\left(W^{s}(x)\right)$ at $x$,

(c) $f\left(W^{u}(x)\right)=W^{u}(f(x))\left(f\left(W^{s}(x)\right)=W^{s}(f(x))\right)$,

(d) if $\Lambda$ is a hyperbolic attractor then $W^{u}(x) \subset \Lambda \subset \Omega(f)$ for all $x \in \Lambda$.

ProOF. It appears in [1].

Definition 1.1. Let $\Lambda$ be a hyperbolic set and $x \in \Lambda$, we will define the $u$-metric in $W^{u}(x)$ and the $s$-metric in $W^{s}(x)$ in the following way:

$d_{u}^{x}(v, z)=\inf \{$ lengths in the metric $d$ on $M$ of the paths contained in $W^{u}(x)$ that begins in $v$ and ends in $z$ and

$d_{s}^{x}(v, z)=\inf \{$ lengths in the metric $d$ on $M$ of the paths contained in $W^{s}(x)$ that begins in $v$ and ends in $z$.

DEFINITION 1.2. Let $\Lambda$ be a hyperbolic set and $x \in \Lambda$, we will define $W_{\varepsilon}^{u}(x)$ the ball of center $x$ and radius $\varepsilon$ in $W^{u}(x)$ in metric $d_{u}^{x}$. Similarly for $W_{\varepsilon}^{s}(x)$.

LEMMA 1.2. Let $\Lambda(f)$ be a hyperbolic set with the periodic points dense in $\Lambda(f)$ and $\varepsilon>0$. Then for any $\delta>0$ there exists an $N>0$ such that $f^{-n}\left(W_{\varepsilon}^{u}(x)\right) \subset W_{\delta}^{u}\left(f^{-n}(x)\right), \forall n>N$.

Proof. Suppose $K$ and $\lambda$ as in the definition of hyperbolicity in the introduction. Given $\delta$ take $n$ such that $K \lambda^{n} \varepsilon<\delta$. Now using (3) on the definition of hyperbolicity, Lemma 1.2 follows.

In the next lemmas we will be interested in the family of pseudo-unstable (pseudo-stable) manifolds that exists for $C^{1}$-structurally stable diffeomorphisms.

We will call $\operatorname{Per}_{j}(f)$ the set of peridoic points of the diffeomorphism $f$ that has stable dimension $j$. Let $\Lambda_{j}(f)$ be the closure of the set $\operatorname{Per}_{j}(f)$. We will use the notation $\mathscr{F}(M)$ to indicate the interior in $\operatorname{Diff}^{1}(M)$ of the set of diffeomorphisms whose periodic points are all hyperbolic.

It is easy to see that all $C^{1}$ structurally stable diffeomorphism is in $\mathscr{F}(M)$.

We point out that Mañe [4] proved that if $f \in \mathscr{F}(M)$ then it follows that $\operatorname{Per}(f)=\Omega(f)$.

We will always suppose in this work the condition $f \in \mathscr{F}(M)$ instead of $f$ $C^{1}$-structurally stable. 
LEMMA 1.3. Let $f \in \mathscr{F}(M)$ with the periodic points dense in $\Lambda_{j} \neq 0$, for some $0<j<\operatorname{dim} M$. Then there exists a continuous splitting $T M / \Lambda_{j}=\bar{E}^{s} \oplus \bar{E}^{u}$ and constants $0<\bar{\lambda}<1, \bar{K}>0$ such that

(a) $(T f) \bar{E}^{s}=\bar{E}^{s}, \operatorname{dim} \bar{E}^{s}=j,(T f) \bar{E}^{u}=\bar{E}^{u}$,

(b) if $x \in \operatorname{Per}_{j}(f)$ and $m$ is the period of $x$, then

$$
\left\|(T f)^{n}\left|\bar{E}_{x}^{s}\left\|<\bar{K} \bar{\lambda}^{m}, \quad\right\|(T f)^{-n}\right| \bar{E}_{x}^{u}\right\|<\bar{K} \bar{\lambda}^{m},
$$

(c) for all $x \in \Lambda_{j}(f)$ and $m \in \mathbf{Z}_{+}$

$$
\left\|(T f)^{m}\left|\bar{E}_{x}^{s}\|\cdot\|(T f)^{-m}\right| \bar{E}_{f^{m}(x)}^{u}\right\|<\bar{K} \bar{\lambda}^{m} .
$$

Proof. It appears in [3].

Let $f \in \mathscr{F}(M)$. Given $0 \leqslant j \leqslant \operatorname{dim} M$ let $\Lambda=\Lambda_{j}(f)$ and $\bar{E}^{s}, \bar{E}^{u}$ be the subbundles of $T M / \Lambda$ given in Lemma 1.3. Denote by $\operatorname{Emb}_{\Lambda}\left(D^{j}, M\right)$ the space of $C^{1}$ embeddings $\beta: D^{j} \rightarrow M$, where $D^{j}=\left\{x \in \mathbf{R}^{j} \mid\|x\|<1\right\}$, such that $\beta(0) \in \Lambda$. Consider $\operatorname{Emb}_{\Lambda}\left(D^{j}, M\right)$ endowed with the $C^{1}$ topology. Then $\pi: \mathrm{Emb}_{\Lambda}\left(D^{j}, M\right) \rightarrow \Lambda$ defined by $\pi(\beta)=\beta(0)$ is a fibration.

LEMMA 1.4. There exist sections $\varphi^{s}: \Lambda \rightarrow \operatorname{Emb}_{\Lambda}\left(D^{j}, M\right), \varphi^{u}: \Lambda \rightarrow$ $\mathrm{Emb}_{\Lambda}\left(D^{\operatorname{dim} M-j}, M\right)$ and $\varepsilon>0$ such that defining

$$
\bar{W}_{\varepsilon}^{s}(x)=\varphi^{s}(x) D_{\varepsilon}^{j}, \quad \bar{W}_{\varepsilon}^{u}(x)=\varphi^{u}(x) D_{\varepsilon}^{\operatorname{dim} M-j},
$$

we have the following properties:

(a) $T_{x} \bar{W}_{\varepsilon}^{s}(x)=\bar{E}_{\underline{x}}^{s}, T_{x} \bar{W}_{\varepsilon}^{u}(x)=\bar{E}_{x}^{u}$.

(b) If we define $\bar{d}_{s}(a, b)=\inf \left\{\right.$ length of $\alpha(t)$, where $\alpha:[0,1] \rightarrow \bar{W}_{\varepsilon}^{s}(x)$ is such that $\alpha(0)=a, \alpha(1)=b\}$ for $x \in \Lambda, a, b \in \bar{W}_{\varepsilon}^{s}(x)$, and define $\bar{B}_{\varepsilon}^{s}(x)=$ $\left\{a \in \bar{W}_{\varepsilon}^{s}(x) \mid \bar{d}_{s}(a, x) \leqslant \varepsilon\right\}$, then $\bar{B}_{s}^{e}(x)=\bar{W}_{e}^{s}(x)$.

(c) There exists a power $g=f^{N}$ of $f$ such that for all $0<\varepsilon_{1}<1$ there exists $0<\varepsilon_{2}<1$ satisfying

$$
g\left(\bar{W}_{e_{2}}^{s}(x)\right) \subset \bar{W}_{\varepsilon_{1}}^{s}(g(x)), \quad \bar{W}_{e_{2}}^{u}(g(x)) \subset g\left(\bar{W}_{\varepsilon_{1}}^{u}(x)\right)
$$

for all $x \in \Lambda$.

REMARK. We call $\overline{W_{\varepsilon_{1}}^{s}}(g(x))$ the set $\bar{B}_{\varepsilon_{1}}^{s}(g(x))$.

ProOF. It appears in [4].

The corresponding properties are true for $\bar{B}_{u}^{\varepsilon}(x)=\bar{W}_{\varepsilon}^{u}(x)$ and $g=f^{-N}$, $N>0$.

These two families of discs will be called, respectively, pseudo-stable and pseudo-unstable manifolds for $x \in \Lambda_{j}(f)$.

REMARK 1. These concepts are "a priori" different from the stable and unstable manifolds defined in Lemma 1.1 .

These two lemmas $(1.3,1.4)$ allow us to use a continuous structure of discs that has some interesting properties. These properties will appear in Lemmas 1.5 through 1.8. This structure however does not have in general the uniform 
property that appears in the hyperbolic case of Lemma 1.2. $f$.

As Lemma 1.3 shows, the $\bar{W}_{e}^{u}(x)$ correspond to the linear invariant part of

REMARK 2. We point out the following invariant properties of the pseudounstable family: for any $\varepsilon>0$ there exist a $\delta>0$ such that $\forall x \in \Lambda_{j}$, $f^{-1}\left(\bar{W}_{\delta}^{u}(x)\right) \subset \bar{W}_{\varepsilon}^{u}\left(f^{-1}(x)\right)$. Suppose now that $p$ is a periodic point, take $\delta$ such that

$$
f^{-j}\left(\bar{W}_{\delta}^{u}(p)\right) \subset \bar{W}_{\varepsilon}^{u}\left(f^{-j}(p)\right), \quad \forall 0<j<\pi(p),
$$

where $\pi(p)$ is the period of $p$. Although in general $f^{-\pi(p)}\left(\bar{W}_{e}^{u}(p)\right) \& \bar{W}_{e}^{u}(p)$, for such $\delta>0$ we have $f^{-\pi(p)}\left(\bar{W}_{\delta}^{u}(p)\right) \subset \bar{W}_{\delta}^{u}(p)$. We use here the uniformity of $\bar{K}$ of Lemma 1.3(b). Remark 2 corresponds to the corollary of Proposition 2.4 in [4].

Lemma 1.5. Let $\varepsilon>0$ be as in Lemma 1.4 and $x \in \operatorname{Per}_{j}(f) \cap \Lambda_{j}$ hyperbolic, then there exist $\varepsilon_{1}>0$ such that $W_{\varepsilon_{1}}^{u}(x)=\bar{W}_{\varepsilon_{1}}^{u}(x)$ (we recall that $W_{\varepsilon_{1}}^{u}(x)$ is the unstable manifold of the hyperbolic periodic point $x$ of size $\varepsilon_{1}$ ).

Proof. Suppose $x$ is fixed for $f$ (otherwise take $n$ such that $f^{n}(x)=x$ ). Let $U$ be the neighborhood of $x$ of size $\varepsilon_{2}$ given by Hartman's theorem (see [1] for reference); that is, $f$ is conjugate to $T f_{x}$ in $U$. By Remark 2 we can take $0<\varepsilon_{1}<\varepsilon_{2}$ such that $f^{-1}\left(\bar{W}_{e_{1}}^{u}(x)\right) \subset \bar{W}_{\varepsilon_{1}}^{u}(x)$. By uniqueness of the $W_{e_{2}}^{u}(x)$, this property just happens for one disc of dimension $j$, the $W_{\varepsilon_{1}}^{u}(x)$ disc. Then it follows that $W_{\varepsilon_{1}}^{u}(x)$ is equal to $\bar{W}_{\varepsilon_{1}}^{u}(x)$.

We point out that this $\varepsilon_{1}$ depends on $x$.

Definition 1.3. For $x, y \in \Lambda_{j}$ we define $\langle x, y\rangle=\bar{W}_{\varepsilon}^{s}(x) \cap \bar{W}_{\varepsilon}^{u}(y)$.

LEMMA 1.6. There exists $\varepsilon>0$ such that if $x \in \Lambda_{j}(f), y \in \Lambda_{j}(f)$ and $d(x, y) \leqslant \varepsilon$ then $\langle x, y\rangle$ is one point and

$$
f(\langle x, y\rangle)=\langle f(x), f(y)\rangle, \quad f^{-1}(\langle x, y\rangle)=\left\langle f^{-1}(x), f^{-1}(y)\right\rangle .
$$

Proof. It appears in [4].

LEMMA 1.7. $\exists \varepsilon_{1}>0, m_{1}>0,0<\bar{\lambda}<1$ and $\bar{K}>0$ such that:

(a) If $x \in \operatorname{Per}_{j}(f)$ has period $\geqslant m_{1}$, then $\bar{d}_{u}\left(f^{n}(x), f^{n}(y)\right)<\varepsilon_{1}$ for all $n>0$ $\left(\bar{d}_{s}\left(f^{n}(x), f^{n}(y)\right) \leqslant \varepsilon_{1}\right.$ for all $\left.n \leqslant 0\right)$ implies $y=x$.

(b) If $x_{i} \in \Lambda_{j}(f), i=1,2, y \in M, N>0$ and $y=\left\langle x_{1}, x_{2}\right\rangle$

$$
\bar{d}_{s}\left(f^{n}\left(x_{1}\right), f^{n}(y)\right) \leqslant \varepsilon_{1}, \quad d_{u}\left(f^{n}\left(x_{2}\right), f^{n}(y)\right)<\varepsilon_{1}
$$

for all $0 \leqslant n \leqslant N$ then:

$$
\frac{\bar{d}_{s}\left(f^{n}\left(x_{1}\right), f^{n}(y)\right)}{\bar{d}_{s}\left(x_{1}, y\right)} \leqslant \bar{K} \bar{\lambda}^{n} \frac{\bar{d}_{u}\left(f^{n}\left(x_{2}\right), f^{n}(y)\right)}{\bar{d}_{u}\left(x_{2}, y\right)}
$$

for all $0 \leqslant n \leqslant N$. 
Proof. It appears in [4].

Lemma 1.8 relates the property that we will really use, and it is a consequence of Lemma 1.7(b). Lemma 1.8 says, informally speaking, that if the images of the positive iterates of $\bar{W}_{\varepsilon}^{u}(x)$ are bounded, then the positive iterates of $\bar{W}_{e}^{s}(x)$ are limited too, for any $x \in \Lambda_{j}$.

LEMMA 1.8. Suppose that for $\varepsilon_{1}>0$ small enough like in Lemma 1.7 and $a, b \in \Lambda_{j}$ such that $a \in \bar{W}_{e_{1}}^{u}(b), \bar{d}_{u}(a, b)=\varepsilon_{1}$ and

$$
\bar{d}_{u_{s}}\left(f^{n}(a), f^{n}(b)\right)<\varepsilon_{1}, \quad \forall n \in \mathbf{Z}_{+},
$$

then $\exists \varepsilon_{2}>0$ such that $\forall y \in \bar{W}_{\varepsilon_{2}}^{s}(b)$,

$$
\bar{d}_{s}\left(f^{n}(b), f^{n}(y)\right)<\varepsilon_{1}, \quad \forall n \in \mathbf{Z}_{+} .
$$

Proof. Let $n_{0}$ be such that $\bar{K} \bar{\lambda}^{n_{0}} \bar{d}_{s}(a, b) / \bar{d}_{u}(a, b)<1$ where $\bar{K}$ and $\bar{\lambda}$ are the constants that appear in Lemma 1.7. Let $\varepsilon_{2}$ be such that

$$
\bar{d}_{s}\left(f^{n}(y), f^{n}(b)\right)<\varepsilon_{1}, \quad \forall n 0 \leqslant n \leqslant n_{0}, \forall y \in \bar{W}_{e_{2}}^{s}(b) .
$$

Then by Lemma 1.7:

$$
\bar{d}_{s}\left(f^{n}(y), f^{n}(b)\right) \leqslant \bar{K} \bar{\lambda}^{n} \frac{\bar{d}_{u}\left(f^{n}(a), f^{n}(b)\right) \cdot \bar{d}_{s}(y, b)}{\bar{d}_{u}(a, b)} \leqslant \varepsilon_{1} \quad \forall n, n>n_{0} .
$$

Then Lemma 1.8 follows.

The next lemma (Lemma 1.9) studies the relation, in the case of an hyperbolic attractor, between the splitting $E^{s}(x) \oplus E^{u}(x)$ (given by hyperbolicity) and the splitting $\bar{E}^{s}(x) \oplus \bar{E}^{u}(x)$ (from Lemma 1.3) for $x$ in the attractor. For the last splitting to make sense of course we consider the attractor contained in $\Lambda_{j}$ for some $0<j<\operatorname{dim} M$.

LEMma 1.9. Let be $\Lambda \subset \Lambda_{j}$ for some $0<j<\operatorname{dim} M, \Lambda$ a hyperbolic attractor for $f \in \mathscr{F}(M)$ and the periodic points dense in $\Lambda$. Then the subspace $E^{u}(x)$ (respectively $\left.E^{s}(x)\right)$ and the subspace $\bar{E}(x)\left(\bar{E}^{s}(x)\right)$ are the same for $x \in \Lambda$.

Proof. We will just consider $E^{u}$ and $\bar{E}^{u}$, because the other case, that is, $E^{s}$ and $\bar{E}^{s}$, is similar. Let $\bar{\varepsilon}$ such that $f^{-n}\left(W_{\bar{\varepsilon}}(\Lambda)\right) \subset W_{\varepsilon}^{u}(\Lambda), \forall n \in \mathbf{Z}_{+}$. The existence of such $\bar{\varepsilon}$ follows from the uniformity of the hyperbolicity (of inequality $\left.\left\|(T f)^{-n} E^{u}(\underline{x})\right\|<K \lambda^{m}, \forall m \in \mathbf{Z}_{+}\right)$. From Lemma 1.5 we have that if $\bar{\varepsilon}$ is small then $\bar{W}_{\bar{\varepsilon}}^{u}(x)=W_{\bar{\varepsilon}}^{u}(x)$, for any $x$ periodic in $\Lambda$. The tangent planes of such manifolds are the same in the periodic points $x$. Now using the fact that the bundles $E_{u}$ and $\bar{E}_{u}$ are continuous, and the periodic points are dense in $\Lambda$, Lemma 1.9 follows.

Lemma 1.10 is a well known fact about hyperbolic attractors. 
LEMMA 1.10. Let $\Lambda$ be a hyperbolic attractor and $c \in \Lambda$ such that $f(c)=c$. Then for all $x, y \in W^{u}(c)$, the sequence $d_{u}^{c}\left(f^{n}(x), f^{n}(y)\right) \rightarrow \infty$ when $n \rightarrow \infty$. Therefore $W^{u}(c)$ is not bounded for the metric $d_{u}^{c}$.

Proof. Let $\alpha:[0,1] \rightarrow W^{u}(c)$ be such that $\alpha(0)=f^{n}(x), \alpha(1)=f^{n}(y)$. As $f^{-n}(\alpha(t))$ is in $\Lambda$, we have:

$$
\begin{aligned}
\int_{0}^{1}\left\|\left(\left(f^{-n} \circ \alpha\right)(t)\right)^{\prime}\right\| d t & =\int_{1}^{0}\left\|(D f)_{f^{-n}(\alpha(t))}^{-n}\left(\alpha^{\prime}(\alpha(t))\right)\right\| d t \\
& \leqslant K \lambda^{n} \int_{0}^{1}\left\|\alpha^{\prime}(\alpha(t))\right\| d t
\end{aligned}
$$

where $K$ and $\lambda$ are the hyperbolic constants for $\Lambda$.

Since $f$ is a diffeomorphism, all paths between $f^{n}(x)$ and $f^{n}(y)$ are $f^{n}$ of some path between $x$ and $y$. Therefore: $d_{u}^{c}(x, y) / K \lambda^{n}<d_{u}^{c}\left(f^{n}(x), f^{n}(y)\right)$.

Then Lemma 1.10 follows and consequently $W^{u}(c)$ is not bounded for $d_{u}^{c}$.

We can give now an idea of the proof of our main result. This program will be carried out in detail in $\$ 2$ and $\$ 3$.

First of all, let us recall the work of Pliss about periodic atrattractors in [5]. (for a $C^{1}$-structurally stable diffeomorphism there are just a finite number of periodic attractors points): he finds $\delta>0$ as small as he wants, and attracting periodic points $p_{i} \in \Lambda_{n}$ (with period $\pi\left(p_{i}\right)$ ) such that there are jumps of constant $k_{i}$-size

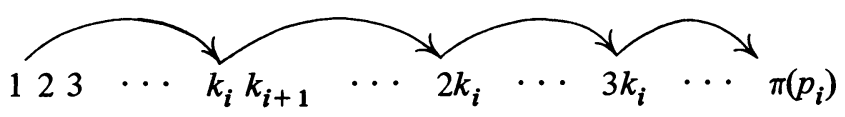

such that $f^{k_{i}}\left(\bar{W}_{\delta}^{s}\left(f^{l k_{i}}\left(p_{i}\right)\right)\right) \subset \bar{W}_{\delta}^{s}\left(f^{(l+1) k_{i}}\left(p_{i}\right)\right)$ is a uniform contraction by $1 / 4$. Here $l>0$ are integers such that $l k_{i} \leqslant \pi\left(p_{i}\right)$. He can make $k_{i}$ and the biggest of such $l$ arbitrarily big, taking $\pi\left(p_{i}\right)$ big enough. Therefore if there exists an infinite number of attracting periodic points, we can take $p_{i}$ with a large number of elements in the above sequence of $k_{i}$-size jumps (that is, the biggest of such $l$, can be taken arbitrarily big). Since $M$ is compact, two of such elements of a sequence must be at a distance less than $\delta / 2$; that is, there exist integers $m_{i}>n_{i}>0$ such that

$$
d\left(f^{m_{i} k_{i}}\left(p_{i}\right), f^{n_{i} k_{i}}\left(p_{i}\right)\right)<\delta / 2
$$

and $m_{i} k_{i}-n_{i} k_{i}<\pi\left(p_{i}\right)$. Well $f^{m_{i} k_{i}-n_{i} k_{i}}$ is a contraction of $\overline{W_{\delta}^{s}}\left(f^{n_{i} k_{1}}\left(p_{i}\right)\right)$ in $\bar{W}_{\delta / 4}^{s}\left(f^{m_{i} k_{i}}\left(p_{i}\right)\right)$; thus $f^{\left(m_{i} k_{i}-n_{i} k_{i}\right) \pi\left(p_{i}\right)}$ has just one fixed point in $W_{\delta}^{s}\left(f^{n_{i} k_{i}}\left(p_{i}\right)\right)$ and different from $p_{i}$ because $m_{i} k_{i}-n_{i} k_{i}<\pi\left(p_{i}\right)$. Thus he reaches a contradiction, and so it follows that the number of periodic attractors is finite.

Remark 3. Pliss in [5] did not explicitly use pseudo-stable manifolds, but the neighborhoods of the points $p_{i}$ that he considered correspond to these manifolds. 
Let us now see our program for proving that if $f \in \mathscr{F}(M)$ then $f$ has only a finite number of hyperbolic attractors.

Suppose to the contrary that there exists an infinite number of hyperbolic attractors in $\Lambda_{j}$. Take $p_{i}$ a periodic point in each attractor, such that $p_{i}$ have minimum period in the attractor.

First we notice that, with the same arguments as in [5], one can get the existence of $k_{i}$-jump sequences as above for the periodic points $p_{i}$ with stable dimension $j, 0<j<\operatorname{dim}(M)$. This fact will be formally stated in Lemma 1.11 .

Another basic fact that is needed, which is Lemma 2.5 proved in $\S 2$, is the following. For some big $N>0, f^{-r}$ is a contraction of $\bar{W}_{\delta}^{u}\left(f^{r}\left(p_{i}\right)\right)$ in $\bar{W}_{\delta}^{u}\left(p_{i}\right)$ for $r>N$; then as in the picture below we will have a new periodic point in $\bar{W}_{\delta}^{u}\left(f^{n_{i} k_{i}}\left(p_{i}\right)\right) \times \bar{W}_{\delta}^{s}\left(f^{n_{i} k_{i}}\left(p_{i}\right)\right)$ if we take $f^{-\left(m_{i} k_{i}-n_{i} k_{i}\right)}$ for $m_{i} k_{i}-n_{i} k_{i}>r$.

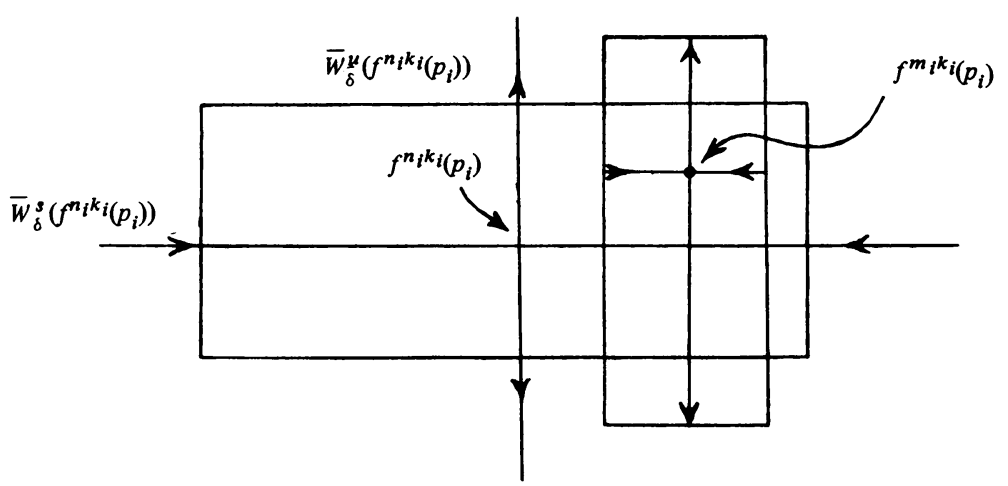

Thus this new periodic point has period less than period of $p_{i}$. Therefore we will have a contradiction if we prove (and we will do it in \$3) that this new periodic point is in the same attractor as $p_{i}$. This is obviously not possible because $p_{i}$ has minimum period in its attractor.

We will finish $\$ 1$ with the new formulation of Pliss' lemma on jump sequences mentioned above. The proof is the same as in [5].

LemMa 1.11. Let $f \in \mathcal{F}(M)$ and a sequence $p_{i} \in \operatorname{Per}_{j}(p)$, that is, $p_{i}$ is a sequence of periodic points with stable dimension $0<j<\operatorname{dim} M$. Suppose that $\pi_{i \rightarrow \infty}\left(p_{i}\right) \rightarrow \infty$, where $\pi\left(p_{i}\right)$ denotes the period of $p_{i}$.

Then $\exists \delta_{0}>0$ such that $\forall \delta<\delta_{0}, \forall N>0, \exists i \in Z_{+}, \exists k_{i}>0, \exists m_{i}>n_{i}>$ 0 such that

(1) $\pi\left(p_{i}\right)>m_{i} k_{i}-n_{i} k_{i}>N>0$

(2) $d\left(f^{m_{i} k_{i}}\left(p_{i}\right), f^{n_{i} k_{i}}\left(p_{i}\right)\right)<\delta / 2$,

(3) $f^{\left(m_{i}-n_{i}\right) k_{i}}$ is a contraction of $\bar{W}_{\delta}^{s}\left(f^{n_{i} k_{i}}\left(p_{i}\right)\right)$ in $\bar{W}_{\delta}^{s}\left(f^{m_{i} k_{i}}\left(p_{i}\right)\right)$, with constant of contracton smaller than $1 / 4$.

In particular $f^{\left(m_{i}-n_{i}\right) k_{i}}\left(\bar{W}_{\delta}^{s}\left(f^{n_{i} k_{i}}\left(p_{i}\right)\right)\right) \subset \bar{W}_{\delta / 4}^{s}\left(f^{m_{i} k_{i}}\left(p_{i}\right)\right)$. 
Proof. Similar to [5].

We point out that the corresponding result for $\bar{W}_{\delta}^{u}$ is true, but unfortunately we cannot obtain simultaneously both results for the same periodic point $p_{i}$.

2. We will consider in this section $f \in \mathscr{F}(M)$ with the periodic points dense in $\Omega(f)$. In the last section we saw that for every $p \in \operatorname{Per}(f)$ there exists a $\delta(p)>0$ such that

$$
\bar{W}_{\delta(p)}^{u}(p)=W_{\delta(p)}^{u} .
$$

Our main purpose in the present section is to obtain a univeral $\delta>0$ so that $\bar{W}_{\delta}^{u}(x)=W_{\delta}^{u}(x)$ for all $x \in A_{H}$, where $A_{H}$ denotes the union of all hyperbolic attractors for $f$ (Lemma 2.7). Moreover we will exhibit an integer $N>0$ so that $f^{-N}$ is a uniform contraction of $\bar{W}_{\delta}^{u}(x)$ in $\bar{W}_{\delta}^{u}\left(f^{-N}(x)\right)$ for all $x \in A_{H}$ and the same $\delta$ (Lemma 2.5).

Of course these facts would be easily verified if we had only a finite number of attractors for $f$. The problem here is that with an arbitrary number of hyperbolic attractors we may not have uniformity in the constants of hyperbolicity.

Let $\varepsilon>0$ be as in Lemma 1.4; such $\varepsilon$ will be used throughout this section in the following way: everywhere we mention $\bar{W}_{r}^{u}(p)$ or $\bar{W}_{r}^{s}(p)$ for some $r>0$, it is implicit that $r<\varepsilon$.

LemMa 2.1. Let $p \in A_{H} \cap \operatorname{Per}(f) \cap \Lambda_{j}$ and $\varepsilon_{0}$ be such that $\forall q \in \bar{W}_{\varepsilon_{0}}^{u}(p)$, $d\left(f^{-n}(q), f^{-n}(p)\right)<\varepsilon, \forall n \geqslant 0 ;$ then $\bar{W}_{\varepsilon_{0}}^{u}(p)=W_{\varepsilon_{0}}^{u}(p)$.

Proof. Let $\pi(p)$ be the period of $p$, therefore by Remark 2 we have $f^{-\pi(p)}\left(\bar{W}_{\varepsilon_{0}}^{u}(p)\right) \subset \bar{W}_{\varepsilon_{0}}^{u}(p)$. We know that there exists by Lemma 1.5 a $\delta(p)>$ 0 such that $W_{\delta(p)}^{u}=W_{\delta(p)}^{u}$.

Suppose to the contrary $\bar{W}_{\varepsilon_{0}}^{u}(p) \neq W_{\varepsilon_{0}}^{u}(p)$, and take $y$ on the boundary of $\bar{W}_{\varepsilon_{0}}^{u}(p) \cap W_{\varepsilon_{0}}^{u}(p)$. Let $V$ be a neighborhood of $y$ in $W_{\varepsilon_{0}}^{u}(p)$. There exist an $n$ such that

$$
f^{-n \pi(p)}(V) \subset W_{\delta(p)}^{u}(p)=\bar{W}_{\delta(p)}^{u}(p) .
$$

As $f^{n \pi(p)}\left(f^{-n \pi(p)}(V)\right) \subset \bar{W}_{\varepsilon_{0}}^{u}$, because $f^{\pi(p)} \bar{W}_{\delta(p)}^{u}(p) \subset \bar{W}_{e_{0}}^{u}(p)$, we have a contradiction with the way we took $y$. Therefore $W_{e_{0}}^{u}(p)=W_{e_{0}}^{u}(p)$.

REMARK 4. The bundle $\bar{E}^{u}$ is uniformly continuous, therefore $\exists \varepsilon_{4}$ such that if $d(x, y)<\varepsilon_{4}$ the angle between $\bar{E}^{u}(x)$ and $\bar{E}^{u}(y)$ is smaller than $1 / 4$. We will consider in the rest of this work the $\varepsilon$ of Lemma 1.3 as being smaller than this $\varepsilon_{4}$.

Lemma 2.2. Let $p \in \operatorname{Per}(f) \cap A_{H} \cap \Lambda_{j}, q \in \Lambda_{j}, \varepsilon$ as in Remark 4 and suppose $\bar{W}_{e}^{u}(q) \subset \Lambda_{j}$, then there exists an $\varepsilon_{5}$ such that if $d(p, q)<\varepsilon_{5}$ then $W^{u}(p) \cap \bar{W}_{e}^{s}(x) \neq \varnothing, \forall x \in \bar{W}_{e}^{u}(q)$. 
Proof. First of all note that there exists an $\varepsilon_{5}<\varepsilon$ such for any surface $V$ of dimension $j$ which is not limited has the following property: If the tangent planes of $V$ and $\bar{E}^{u}(y), \forall y \in \bar{W}_{\varepsilon}^{u}(q)$ are smaller than $1 / 4$ and there exist $z \in V, d(z, q)<\varepsilon_{5}$, then $V$ is the graph of some function of $\bar{W}_{e}^{u}(q)$ to $\mathbf{R}$. Therefore $V \cap \bar{W}_{\varepsilon}^{s}(x) \neq \varnothing$ for any $x \in \bar{W}_{\varepsilon}^{u}(q)$ such that $d(x, q)<\varepsilon$. We just have to show that $W^{u}(p), \forall p \in \operatorname{Per} f \cap A_{H} \cap \Lambda_{j}$, satisfies the conditions of $V$. Remember that by Lemma $1.10, W^{u}(p)$ is not limited and by Lemma $1.1(\mathrm{~d}), W^{u}(p) \subset \Lambda_{j}$. Now by Lemma $1.9 \bar{E}^{u}(z)=E^{u}(z)$, then for $y \in \bar{W}_{\varepsilon}^{u}(q)$ and $z \in W_{\varepsilon}^{u}(p)$ the angles between $\bar{E}^{u}(y)$ and $\bar{E}^{u}(z)$ are smaller than $1 / 4$ by the way we took $\varepsilon$ (see Remark 4). Therefore Lemma 2.2 is true.

REMARK 5. Let $\varepsilon_{5}$ be fixed satisfying the conditions of Lemma 2.2. Then there exists a $C>0$ such that for $p \in \operatorname{Per}_{j} f \cap A_{H} \cap \Lambda_{j}, d(p, q)<\varepsilon_{5}$, the distance between $x$ and $y$ in the same connected component of $W^{u}(p) \cap$ $\left(\bar{W}_{\varepsilon}^{s}(q) \times \bar{W}_{\varepsilon}^{u}(q)\right)$ is uniformly bounded by $C$. The reason is that the angles between $\bar{E}^{u}(x)$ and $\bar{E}^{u}(z)$ are limited for all $x \in W^{u}(p)$ and $z \in \bar{W}_{\varepsilon}^{u}(q)$, $d(p, x) \leqslant \varepsilon$.

We will suppose in the rest of this work the $\varepsilon$ of Remark 4 smaller than this fixed $\varepsilon_{5}$.

The following Lemmas 2.3 and 2.4 are just for periodic points but they are the central point of $\$ 2$.

The main purpose of this section, Lemmas 2.5 and 2.7, will follow by the continuity of $\bar{W}_{\varepsilon}^{u}(x)$ in $\Lambda_{j} \cap A_{H}$.

In the next lemma (Lemma 2.3) we will obtain a property that is very easy to prove for just one hyperbolic attractor (Lemma 1.2).

The problem here is that we could have an infinite number of attractors and therefore the constants $K$ can be arbitrarily close to 0 .

LEMMA 2.3. $\exists \varepsilon_{0}>0$ such that $\forall \varepsilon_{6}, 0<\varepsilon_{6}<\varepsilon_{0}, \exists \delta>0$ such that $f^{-n}\left(\bar{W}_{\delta}^{u}(p)\right) \subset \bar{W}_{\varepsilon_{6}}^{u}\left(f^{-n}(p)\right)$ for all $p \in A_{H} \cap \operatorname{Per}_{j}(f) \cap \Lambda_{j}, \forall n \in \mathbf{Z}_{+}$.

Proof. Let $\varepsilon_{0}=\varepsilon_{5}$ as in Remark 5 after Lemma 2.2. For $p \in \Lambda_{j}$ we define $S_{p}=\left\{\alpha \in \bar{W}_{\varepsilon_{6}}^{u} \mid d\left(f^{-n}(p), f^{-n}(\alpha)\right)<\varepsilon_{6}, \quad \forall n \in \mathbf{Z}_{+}\right\}$and $\delta_{p}=$ $d\left((p, 0), \bar{W}_{\varepsilon_{6}}^{u}(p)-S p\right), \forall \varepsilon_{6}<\varepsilon$.

Notice that by Lemma 1.3(b) we have that if $\pi(p)$ is big enough $f^{-\pi(p)}\left(\bar{W}_{\delta_{p}}^{u}(p)\right) \subset \bar{W}_{\delta_{p}}^{u}(p), \forall p \in \operatorname{Per}(f) \cap \Lambda_{j}$. Thus $\delta_{p}>0$ always. Also if $x \in \bar{W}_{\delta_{p}}^{u}(p)$ then $d\left(f^{-n}(p), f^{-n}(x)\right) \leqslant \varepsilon_{6}$ for all $n>0$.

Suppose to the contrary there exist $\delta_{n}=\delta_{p_{n}} \rightarrow 0, p_{n} \in \operatorname{Per}_{j}(f) \cap A_{H} \cap \Lambda_{j}$. Let $q_{n} \in \Lambda_{j} \cap \bar{W}_{\delta_{n}}^{u}\left(p_{n}\right), 0<m_{n} \leqslant \pi\left(p_{n}\right), d\left(f^{-m_{n}}\left(q_{n}\right), f^{-m_{n}}\left(p_{n}\right)\right)=\varepsilon_{6}$. It is clear that $m_{n} \rightarrow \infty$ because as $f$ is $C^{1}$ and $M$ is compact, the derivative of $f$ is uniformly bounded.

Let $a=\lim f^{-m_{n}}\left(q_{n}\right) \in \Lambda_{j}, \quad b=\lim f^{-m_{n}}\left(p_{n}\right) \in \Lambda_{j}$. We will show that $d\left(f^{n}(a), f^{n}(b)\right)<\varepsilon_{6}, \forall n \in \mathbf{Z}_{+}$. 
Let $B_{\Delta}(x)=\{y \in M \mid d(x, y)<\Delta\}$. Suppose to the contrary that $\exists n_{0}>0$ such that $d\left(f^{n_{0}}(a), f^{n_{0}}(b)\right)>\varepsilon_{6}$.

Let $\Delta_{1}$ such that $\forall x \in B_{\Delta_{1}}\left(f^{n_{0}}(a)\right), \forall y \in B_{\Delta_{1}}\left(f^{n_{0}}(b)\right)$ we have $d(x, y)>\varepsilon_{6}$. Let now $\Delta_{2}$ be such that $\forall x \in B_{\Delta_{2}}(a), \forall y \in B_{\Delta_{2}}(b)$ we have

$$
f^{n_{0}}(x) \in B_{\Delta_{1}}\left(f^{n_{0}}(a)\right), \quad f^{n_{0}}(y) \in B_{\Delta_{1}}\left(f^{n_{0}}(b)\right) .
$$

Let $p_{n}, q_{n}$ be such that $d\left(f^{-m_{n}}\left(q_{n}\right), a\right)<\Delta_{2}, d\left(f^{-m_{n}}\left(p_{n}\right), b\right)<\Delta_{2}, m_{n}>n_{0}$. Thus

$$
d\left(f^{-m_{n}+n_{0}}\left(p_{n}\right), f^{-m_{n}+n_{0}}\left(q_{n}\right)\right)>\varepsilon_{6},
$$

but this is a contradiction with the fact that $q_{n} \in \bar{W}_{\delta_{p_{n}}}^{u}\left(p_{n}\right)$. Therefore $\forall n \in$ $\mathbf{Z}_{+}, d\left(f^{n}(a), f^{n}(b)\right)<\varepsilon_{6}$.

We point out that by the way we take $a$ and $b$ we have that $a \in \bar{W}_{\varepsilon_{6}}^{u}(b)$. Let now $c \in A_{H}$ some periodic point of the sequence $f^{-m_{n}}\left(p_{n}\right)$. By Lemma 2.2 if $c$ is close enough of $b$ then we have the following: there exists an $a_{1}$ in the intersection $\bar{W}_{\varepsilon_{6}}^{s}(a) \cap W^{u}(c)$ and a $b_{1}$ in the intersection of $\bar{W}_{\varepsilon_{6}}^{s}(b) \cap$ $W^{u}(c)$.

Now by Lemma 1.8 if $d\left(a_{1}, a\right)<\varepsilon_{2}$ and $d\left(b_{1}, b\right)<\varepsilon_{2}$ for some $\varepsilon_{2}>0$ then $d\left(f^{n}\left(a_{1}\right), f^{n}\left(b_{1}\right)\right)<\varepsilon_{6}, \forall n \in \mathbf{Z}_{+}$. It is clear that we can take $a_{1}$ near $a$ and $b_{1}$ near $b$ by taking $c$ near $b$. Thus $d\left(f^{n}\left(a_{1}\right), f^{n}\left(b_{1}\right)\right)<\varepsilon_{6}$ for all $n>0$ and $a_{1}, b_{1} \in W^{u}(c)$.

We will show finally that this will imply that $d_{u}^{c}\left(f^{n}\left(a_{1}\right), f^{n}\left(b_{1}\right)\right)$ is limited, and therefore by Lemma 1.10 we will have a contradiction. The distance $d_{u}^{c}\left(f^{n}\left(a_{1}\right), f^{n}\left(b_{1}\right)\right)$ is limited by $C$ (see Remark 5 of Lemma 2.2) because

$$
d\left(f^{n}\left(a_{1}\right), f^{n}\left(b_{1}\right)\right) \leqslant \varepsilon_{6} \leqslant \varepsilon_{5} .
$$

Therefore with this contradiction we conclude that Lemma 2.3 is true.

LEMMA 2.4. Let $\varepsilon_{0}$ be as in Lemma 2.3. There exists an $0<\varepsilon_{7}<\varepsilon_{0}$ such that $F_{n}(p)=\operatorname{diam} f^{-n}\left(\bar{W}_{\varepsilon_{7}}^{u}(p)\right)$ converges uniformly to zero for $p \in A_{H} \cap \operatorname{Per}(f)$ $\cap \Lambda_{j}$ when $n \rightarrow \infty$.

Proof. We have to prove the following: $\forall \varepsilon_{3}>0, \exists N>0$ such that for all $p$ in $A_{H} \cap \operatorname{Per}_{j}(f) \cap \Lambda_{j}, \forall n>N, f^{-n}\left(\bar{W}_{\varepsilon_{7}}^{u}(p)\right) \subset \bar{W}_{\varepsilon_{3}}^{u}\left(f^{-n}(p)\right)$.

Let $\varepsilon_{7}>0$ be such that $f^{-n}\left(\bar{W}_{\varepsilon_{7}}^{u}(p)\right) \subset \bar{W}_{\varepsilon_{0}}^{u}\left(f^{-n}(p)\right), \forall n \in \mathbf{Z}_{+}$as in Lemma 2.3. Given $\varepsilon_{3}>0$ and $\varepsilon_{3}<\varepsilon_{7}$, again by Lemma 2.3 there exists $\delta>0$ such that $f^{-n}\left(\bar{W}_{\delta}^{u}(p)\right) \subset \bar{W}_{\varepsilon_{3}}\left(f^{-n}(p)\right), \forall n \in \mathbf{Z}_{+}, \forall p \in\left(\Lambda_{j} \cap \operatorname{Per}_{j}(f) \cap A_{H}\right)$.

Suppose to the contrary that $\exists n_{k}$ sequence in $\mathbf{Z}_{+} n_{k} \rightarrow \infty$ such that $F_{n_{k}}\left(p_{k}\right)>\varepsilon_{3}$ for some fixed $\varepsilon_{3}$. Let $q_{k} \in \bar{W}_{\varepsilon_{7}}^{u}\left(p_{k}\right)$ be such that

$$
d\left(f^{-n_{k}}\left(q_{k}\right), f^{-n_{k}}\left(p_{k}\right)\right)=\varepsilon_{3} \text {. }
$$

It is clear that $d\left(f^{-j}\left(q_{k}\right), f^{-j}\left(p_{k}\right)\right) \geqslant \delta, \forall j, 0<j<n_{k}$, by the way we took $\delta$.

Let now $a=\lim f^{-\bar{n}_{k}}\left(q_{k}\right)$ and $b=\lim f^{-\bar{n}_{k}}\left(p_{k}\right)$ where $\bar{n}_{k}$ is the smallest 
integer larger than $\frac{1}{2} n_{k}$. Note that by the way we took $\varepsilon_{7}, d\left(f^{-j}\left(q_{k}\right)\right.$, $\left.f^{-j}\left(p_{k}\right)\right)<\varepsilon_{0}, \forall j, 0<j<n_{k}$.

Using the same arguments of Lemma 2.3 we have that $\delta<d\left(f^{n}(b), f^{n}(a)\right)$ $<\varepsilon_{0}$.

Again (as in Lemma 2.3) by remarks of Lemma 2.2 we conclude that $d_{c}^{u}\left(f^{n}\left(a_{1}\right), f^{n}\left(b_{1}\right)\right)$ is bounded for $n \in \mathbf{Z}_{+}, c$ as Lemma 2.3. As $c \in A_{H}$ this last sentence is in contradiction with Lemma 1.10. Therefore our first assumption in the proof of this lemma is false, and consequently Lemma 2.4 is true.

LEMMA 2.5. Let $\varepsilon_{0}, \varepsilon_{7}$ and $F_{n}(p)$ as in Lemma 2.4, then $F_{n}(p)$ converges uniformly to zero when $n \rightarrow \infty$ for all $p \in A_{H} \cap \Lambda_{j}$.

Proof. Suppose to the contrary that Lemma 2.5 is false, therefore $\exists \varepsilon_{3}>0$, $\exists x_{k} \in A_{H} \cap \Lambda_{j}, \exists y_{k} \in \bar{W}_{\varepsilon_{7}}^{u}(x)$ and $n_{k} \rightarrow \infty$, such that

$$
d\left(f^{-n_{k}}(x), f^{-n_{k}}(y)\right)=2 \varepsilon_{3} .
$$

For each $k$, take $p_{k} \in A_{H} \cap \operatorname{Per}_{j} f \cap \Lambda_{j}$ and $q_{k} \in \bar{W}_{\varepsilon_{7}}^{u}\left(p_{k}\right)$ sufficiently close respectively to $x$ and $y$, using the continuity of the pseudo-unstable families in $\Lambda_{j}$. If these distances $d\left(x, p_{k}\right)$ and $d\left(y, q_{k}\right)$ are small, then $d\left(f^{-n_{k}}\left(p_{k}\right), f^{-n_{k}}\left(q_{k}\right)\right)>\varepsilon_{3}$. As $n_{k} \rightarrow \infty$ this last sentence is in contradiction with Lemma 2.4. Therefore Lemma 2.5 is true.

LEMMA 2.6. There exists a constant $\delta$ such that $W_{0}^{u}(p)=\bar{W}_{\delta}^{u}(p)$ for all $p \in A_{H} \cap \operatorname{Per}(f) \cap A_{j}$.

Proof. Let $\delta$ be obtained as in Lemma 2.3 for the $\varepsilon_{6}=\varepsilon_{0}<\varepsilon$.

As we have seen in Lemma $2.3, f^{-n}\left(\bar{W}_{\delta}^{u}(p)\right) \subset \bar{W}_{\varepsilon}^{u}\left(f^{-n}(p)\right), \forall n \geqslant 0$. Therefore $\forall p \in \operatorname{Per} f \cap A_{H} \cap \Lambda_{j}, f^{-\pi(p)}\left(\bar{W}_{\delta}^{u}(p)\right) \subset \widetilde{W}_{\delta}^{u}(p)$ by Remark 2 before Definition 1.3. We conclude then by Lemma 2.1 that $W_{\delta}^{u}(p)=$ $\bar{W}_{\delta}^{u}(p)$.

LEMMA 2.7. The constant $\delta$ of Lemma 2.6 is such that $W_{\delta}^{u}(p)=\bar{W}_{\delta}^{u}(p)$, $\forall p \in A_{H} \cap \Lambda_{j}$.

Proof. The points $p \in \operatorname{Per} f \cap A_{H} \cap \Lambda_{j}$ are dense in each attractor in $A_{H} \cap \Lambda_{j}$. Therefore by Lemma 2.6, and by the continuities of the pseudounstable manifold family in $\Lambda_{j}$ and unstable manifold family in an attractor in $A_{H}$, we have that for any $q \in A_{H} \cap \Lambda_{j}, W_{\delta}^{u}(q)=\bar{W}_{\delta}^{u}(q)$.

REMARK 6. We point out that if we take $\varepsilon_{3}<\delta, \varepsilon_{3}<\varepsilon_{7} / 4$ and $N>0$ as in Lemma 2.5, then we will have $f^{-N}$ a contraction in $W^{u}(p)$ for all $p \in A_{H} \cap$ $\Lambda_{j}$. In this case the constant of contraction is smaller than 1/4. The reason is that any $q \in W^{u}(p)$ is in $\Lambda_{j} \cap A_{H}$, and therefore $W_{\delta}^{u}(q)=\bar{W}_{\delta}^{u}(q)$. Thus as $f^{-N}\left(\bar{W}_{\varepsilon_{7}}^{u}(q) \subset \bar{W}_{\varepsilon_{3}}^{u}\left(f^{-N}(q)\right), f^{-N}\right.$ is a contraction of $W^{u}(p)$ in $W^{u}\left(f^{-N}(p)\right)$, $\forall p \in A_{H} \cap \Lambda_{j}$. 
3.

THEOREM. If $M$ is a compact $C^{\infty}$ manifold without boundary and $f$ is $C^{1}$-structurally stable, then the number of hyperbolic attractors is finite.

Proof. It is enough to prove that the number of hyperbolic attractors for $f$ in $\Lambda_{j}$ is finite. We point out that each attractor is completely contained in some $\Lambda_{j}$, and there is just a finite number of $\Lambda_{j}$.

All we will need for the proof is that $f \in \mathscr{F}(M)$ and that the periodic points are dense in $\Omega(f)$.

For each hyperbolic attractor $\Lambda$ in $\Lambda_{j}$ we will define

$$
i(\Lambda)=\min \left\{\operatorname{per}(x) \mid x \in \operatorname{Per}_{j}(f) \cap \Lambda \cap \Lambda_{j}\right\}
$$

Suppose to the contrary that there exists an infinite number of attractors, so that $i(\Lambda)$ is not bounded as $\Lambda$ varies in $A_{H}$ (all periodic points are hyperbolic).

Take $p_{n}$ a periodic point with minimum period in each attractor in $\Lambda_{j}$. It is clear that $\pi\left(p_{n}\right) \rightarrow \infty$.

Now using Lemma 1.11 for $\left(p_{n}\right)$, and $\delta / 2$ ( $\delta$ as in Lemma 2.7 we will have the following: $\exists p_{i}$ in the sequence $\left(p_{n}\right)$ such that $f^{\left(m_{i}-n_{i}\right) k_{i}}$ is a contraction of $\bar{W}_{\delta}^{s}\left(f^{n_{i} k_{i}}\left(p_{i}\right)\right)$ in $\bar{W}_{\delta}^{s}\left(f^{m_{i} k_{i}}\left(p_{i}\right)\right)$ with constant of contraction smaller than $1 / 4$. As we have seen in Lemma $1.11,\left(m_{i}-n_{i}\right) k_{i}$ can be taken as large as we want,

$$
d\left(f^{m_{i} k_{i}}\left(p_{i}\right), f^{n_{i} k_{i}}\left(p_{i}\right)\right)<\delta / 2
$$

and $0<\left(m_{i}-n_{i}\right) k_{i}<\pi\left(p_{i}\right)$.

If $\left(m_{i}-n_{i}\right) k_{i}$ is large enough then by Lemma 2.5 , we will have that $f^{-\left(m_{i}-n_{i}\right) k_{i}}$ is a contraction of $\bar{W}_{4 \delta}^{u}\left(f^{m_{i} k_{i}}\left(p_{i}\right)\right)$ with constant of contraction smaller than $1 / 4$.

Let $\Lambda$ be the hyperbolic attractor such that $p_{i} \in \Lambda$. Now let

$$
S=\bar{W}_{\delta}^{s}\left(f^{n_{i} k_{i}}\left(p_{i}\right)\right) \cap\left(\bigcup_{x \in \Lambda} W_{\delta}^{u}(x)\right) .
$$

Now we will define the map $g: S \rightarrow S$ by

$$
g(x)=\bar{W}_{\delta}^{s}\left(f^{n_{i} k_{i}}\left(p_{i}\right)\right) \cap W_{\delta}^{u}\left(f^{\left(m_{i}-n_{i}\right) k_{i}}(x)\right) .
$$

This map is well defined by Lemma 2.7. We point out that $S$ is a complete metric space for the induced metric of $\bar{W}_{\delta}^{s}\left(f^{n_{i} k_{i}}\left(p_{i}\right)\right)$. As we have seen by Lemma $2.5, g$ is a contraction in $S$. Take $x$ the fixed point of $g$ and we will have that $f^{-\left(m_{i}-n_{i}\right) k_{i}}$ is a contraction of $W_{4 \delta}^{u}(x)$ in $W_{\delta}^{u}(x)$. Therefore there exists a periodic point with period $\left(m_{i}-n_{i}\right) k_{i}$ in $\Lambda$, but this is impossible because $\left(m_{i}-n_{i}\right) k_{i}<\pi\left(p_{i}\right)$.

The conclusion is that there exists only a finite number of attractors in $\Lambda_{j}$. As there is a finite number of $\Lambda_{j}$ and their union is the whole $\Omega(f)$, we conclude that there exists just a finite number of hyperbolic attractors. 


\section{REFERENCES}

1. M. Hirsch and C. Pugh, Stable manifolds and hyperbolic sets, Proc. Sympos. Pure Math., vol. 14, Amer. Math. Soc., Providence, R. I., 1970, pp. 133-164.

2. J. Palis and S. Smale, Structural stability theorems, Proc. Sympos. Pure Math., vol. 14, Amer. Math. Soc., Providence, R. I., 1970, pp. 223-231.

3. R. Mañé, Expansive diffeomorphisms, Dynamical Systems, Lecture Notes in Math., vol. 468, Springer-Verlag, Berlin and New York, 1975, pp. 162-174.

4. Contributions to the stability conjecture, Topology (to appear).

5. V. A. Pliss, $A$ hypothesis due to Smale, Differential Equations 8 (1972), 203-214.

6. The location of separatrices of periodic saddle-point motion of second order differential equations, Differential Equations 7 (1971), 906-927.

7. C. Pugh, The closing lemma, Amer. J. Math. 85 (1967), 956-1009.

8. J. Robbin, $A$ structural stability theorem, Ann. of Math. 94 (1971), 447-493.

9. C. Robinson, Structural stability of $C^{\prime}$ flows, Lecture Notes in Math., vol. 468, SpringerVerlag, Berlin and New York, 1975, pp. 262-277.

10. , Structural stability of $C^{1}$ diffeomorphisms, J. Differential Equations 22 (1976), 28-73.

11. S. Smale, Differential dynamical systems, Bull. Amer. Math. Soc. 73 (1976), 747-817.

Instituto de Matemítica, UFRGS, Rua SARmento Letre, 425, $3^{\circ}$ ANDar, 90000 Porto ALEGRE, RS BRASIL 